Revue des patrimoines

$12 \mid 2009$

Le patrimoine religieuX des $\mathrm{XIX}^{\mathrm{e}}$ et $\mathrm{XX}$ siècles $-2^{\mathrm{e}}$ partie

\title{
La Clarté-Dieu (Orsay, Essonne) : simplicité, sobriété, beauté
}

Annabel Rémy

\section{(2) OpenEdition}

Journals

Édition électronique

URL : http://journals.openedition.org/insitu/5394

DOI : 10.4000/insitu.5394

ISSN : 1630-7305

Éditeur

Ministère de la culture

Référence électronique

Annabel Rémy, «La Clarté-Dieu (Orsay, Essonne) : simplicité, sobriété, beauté », In Situ [En ligne],

12 | 2009, mis en ligne le 03 novembre 2009, consulté le 19 avril 2019. URL : http://

journals.openedition.org/insitu/5394; DOI : 10.4000/insitu.5394

Ce document a été généré automatiquement le 19 avril 2019

\section{cc) $(1) \odot$}

In Situ Revues des patrimoines est mis à disposition selon les termes de la licence Creative Commons Attribution - Pas d'Utilisation Commerciale - Pas de Modification 4.0 International. 


\section{La Clarté-Dieu (Orsay, Essonne) : simplicité, sobriété, beauté}

\section{Annabel Rémy}

\section{Historique}

Figure 1

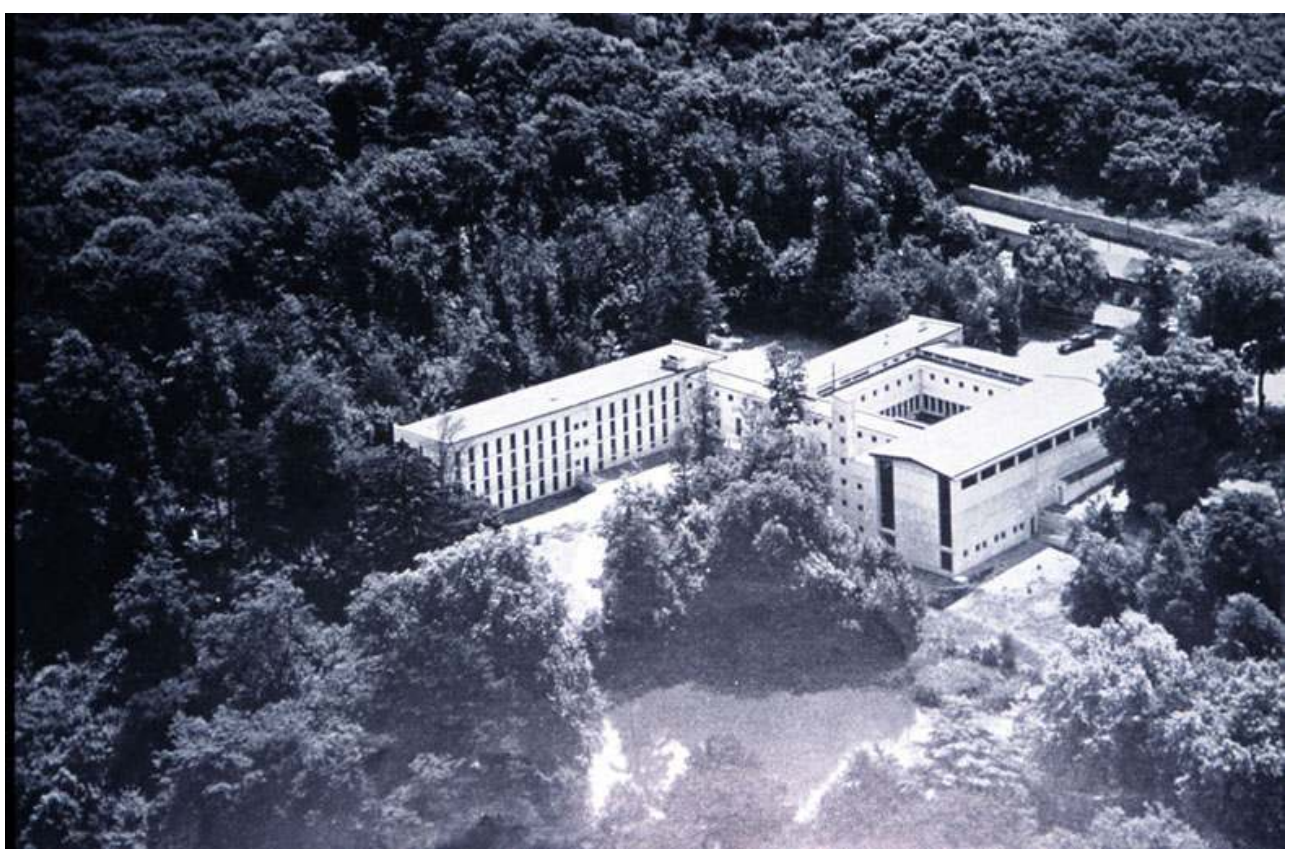

Vue aérienne du couvent de La Clarté-Dieu, photo ancienne (fonds d'archives de La Clarté-Dieu).

Repro. Annabel Rémy, 2008.

(c) Annabel Rémy.

$\mathrm{Au}$ lendemain de la Seconde Guerre mondiale, les frères franciscains, en Ile-de-France, ne disposaient pour tout noviciat que du bâtiment vétuste et peu adapté de Champfleury à 
Poissy (Yvelines). Les dommages de guerre touchés en réparation de la destruction de leur couvent d'Amiens leur permirent d'envisager la construction d'un nouvel ensemble conventuel, destiné à devenir un centre d'études de théologie. Construit à une époque où la vocation religieuse n'était pas en recul, le nouveau couvent devait pouvoir accueillir 80 religieux : 50 frères étudiants, 15 pères, 15 frères convers (fig. $n^{\circ} 1$ ).

2 Un grand terrain arboré et pourvu d'une pièce d'eau fut acquis à 20 kilomètres au sud de Paris, sur la commune d'Orsay. Le père Pol de Léon chargea alors deux frères, frère Alphonse (ingénieur agronome) et frère Ludovic (docteur en droit), assistés d'un jeune architecte proche des franciscains, Emmanuel Besnard-Bernadac (1924- ), de rédiger un programme à l'attention des architectes et de sélectionner ces architectes.

3 Le programme rédigé ${ }^{1}$ est d'une précision étonnante. Ses 97 pages décrivent avec minutie le nombre de pièces nécessaires, leurs connexions, leur usage, leur mobilier. Les deux notes préliminaires, la première sur «l'esprit qui doit présider à une construction franciscaine ", la seconde sur "le caractère religieux dans l'architecture", sont fondamentales pour la conception et la réalisation de cette construction. Elles adoptent les idées régulièrement développées par le père Régamey dans la revue l'Art Sacré, incitant à un renouveau puisant dans l'art et les techniques contemporaines.

On peut s'étonner que pour un projet de cette ampleur, les pères franciscains aient choisi de jeunes architectes, certes brillants élèves (Xavier Arsène-Henry [1919- ] avait été reçu second au concours du Grand prix de Rome), et prometteurs dans leur collaboration avec Louis Arretche (1905-1991), mais n'ayant que peu de réalisations à leur actif, et la plupart à l'extérieur du territoire national (Conakry, Agadir, Djidjelli...). Le frère Alphonse avait en fait " pressenti » plusieurs architectes et mené une enquête sur leur compte, se faisant également conseiller par le père Régamey (directeur de la revue l'Art Sacré). Les architectes en question étaient Marcel et son fils Jacques Page, Pierre Vago (1910-2002), Novarina (1907-2002), Rémy Le Caisne, Dubuisson (1914- ), et les frères Arsène-Henry (Xavier et Luc [1924- ]), sur conseil d'un ami architecte des franciscains, Emmanuel Besnard-Bernadac. Pour chacun des candidats, le frère Alphonse a dressé des fiches analysant la morale, les méthodes de travail, les références, la possibilité de collaboration avec les frères (le souci d'œuvre spirituelle).

5 Les frères Arsène-Henry sont choisis pour leur sensibilité à la religion catholique, pour leur liens avec l'ingénieur Wladimir Bodiansky, parce qu'Emmanuel Besnard-Bernadac les recommande aux franciscains, et parce qu'ils acceptent les honoraires les moins élevés des taux légaux de l'époque. Le 5 janvier 1953, les frères Arsène-Henry sont informés par courrier qu'ils sont retenus pour le projet, à condition qu'ils fassent équipe avec Emmanuel Besnard-Bernadac.

Il est intéressant de comparer la conception du projet et le choix des architectes sur ce chantier d'Orsay avec les deux autres grands chantiers monastiques de cette décennie : le couvent des dominicains de Lille (1952-1966) construit par Pierre Pinsard (1906-1988) ${ }^{2}$ et le couvent de La Tourette à Eveux-sur-L'Arbresle construit par Le Corbusier (1887-1965) (1956-1961), tous deux protégés au titre des Monuments historiques. À Lille, le père Brous avait contacté Pierre Pinsard après avoir vu sa réalisation pour les dominicains rue de la Glacière à Paris ( $13^{\mathrm{e}}$ arr.) et après avoir refusé de contacter Le Corbusier, comme le père Couturier le lui avait suggéré, ne voulant pas d'un artiste ni d'un concept. À Eveux, le père Couturier a justement choisi Le Corbusier parce que c'était un artiste qui bien que n'étant pas croyant avait « suffisamment le sens du mystère $»^{3}$ pour concevoir un édifice religieux. 
7 Dix-huit mois seront consacrés à la conception du projet, les franciscains insistant pour que tout soit prévu avant que la première pierre ne soit posée, ceci afin d'éviter des travaux supplémentaires, toujours coûteux. Tous les documents de conception et de réalisation du couvent sont conservés dans les archives de La Clarté-Dieu (in situ). Un premier projet est rendu le 28 janvier 1953. Dès cette date, la forme générale du couvent, l'orientation sur le terrain sont fixées et ne seront qu'affinées au fil du temps et des plans rendus. Ce qui change en revanche, ce sont les matériaux choisis. Initialement prévu en moellons de pierre et en béton, le projet évolue vers un voile en béton de ciment blanc (fig. $\left.n^{\circ} 2, n^{\circ} 3\right)$.

Figure 2

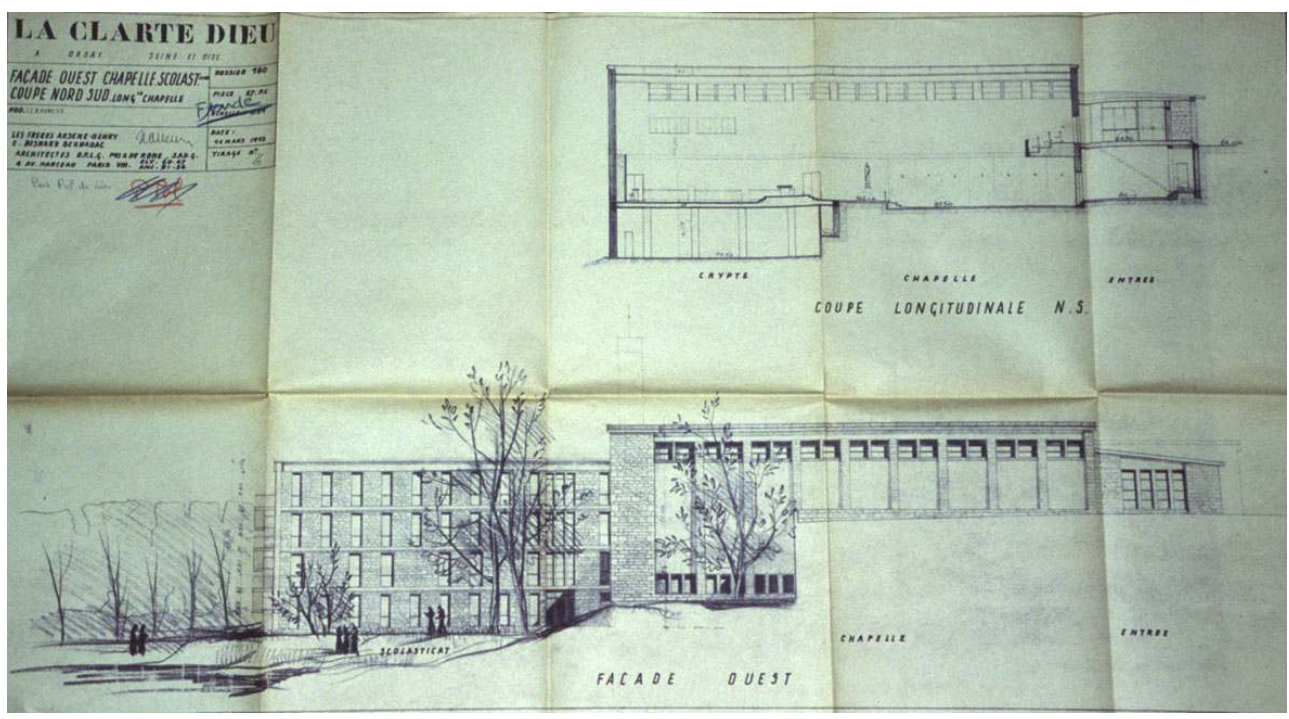

Élévation de la façade ouest du couvent (fonds d'archives de La Clarté-Dieu). Si le dessin correspond bien à ce qui sera exécuté, en revanche le matériau change : les architectes renoncent aux moellons qui figurent sur ce plan. Repro. Annabel Rémy, 2008.

(c) Annabel Rémy. 
Figure 3

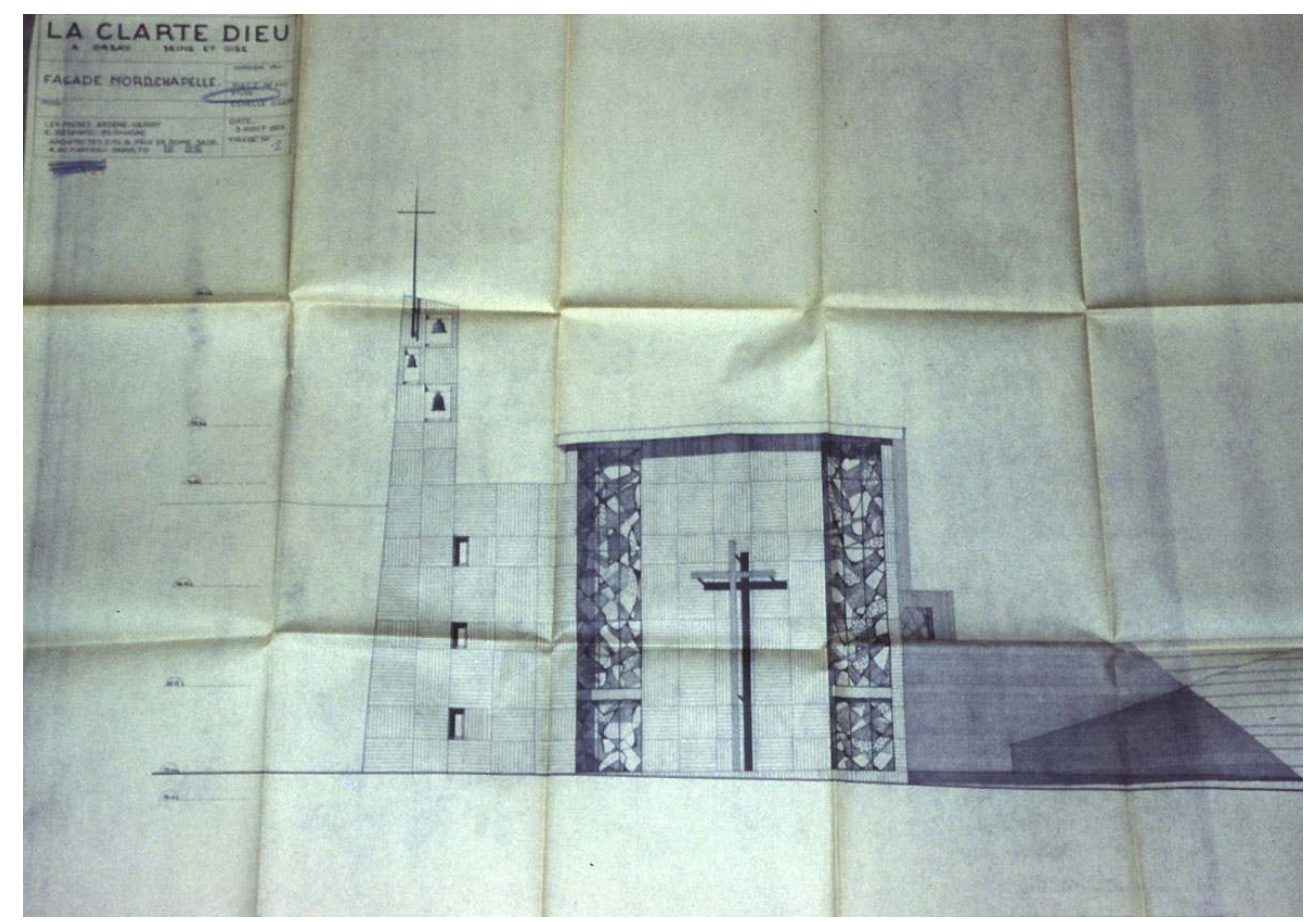

Élévation de la façade nord de la chapelle en voile de béton de ciment blanc (fonds d'archives de La Clarté-Dieu). Repro. Annabel Rémy, 2008.

(C) Annabel Rémy.

Les études et carottages du terrain, effectués à la demande des architectes par l'entreprise Bachy sous le contrôle du bureau d'étude Atbat (dans lequel travaille Wladimir Bodiansky), permettent d'identifier une nature de sols assez instable (sable, blocs de grès épars, présence d'eau). Les fondations sont faites sur pieux de béton coulés et moulés dans le sol, à une profondeur moyenne de 7 mètres. D'une largeur de 50 centimètres de diamètre, ils agissent par appui sur le bon sol et par frottement sur les terrains traversés (selon le système Franck). L'assiette de base des bâtiments est constituée de 350 pieux dont les têtes sont reliées par des longrines.

La pose de la première pierre a lieu officiellement le 9 janvier 1955.

Les bétons ont été mis en œuvre avec beaucoup de soin par l'ingénieur Wladimir Bodiansky (des goulottes étaient insérées dans les coffrages et remontées au fur et à mesure de la coulée du béton pour éviter toute dissociation des éléments constituants), et sont aujourd'hui dans un parfait état de conservation: les arêtes des piliers du cloître sont restées vives, et un seul éclatement du béton a été constaté - sur la paroi de l'escalier extérieur au nord du scolasticat.

11 La couverture des bâtiments est faite en cuivre, ce qui représentait certes un coût élevé mais devait garantir une étanchéité parfaite pour quelques décennies. Elle s'est révélée être le « talon d'Achille » de l'aile du scolasticat, tant par une malfaçon lors de la pose que par un défaut de conception : la pente du toit est trop faible pour ce type de revêtement, $9 \%$ au lieu des $12 \%$ préconisés par la Société industrielle et commerciale du cuivre.

Le bâtiment est terminé et inauguré le 4 juillet 1956, consacré le 30 septembre de la même année et, dès le mois d'octobre, la clôture est effective. Dès son inauguration, le couvent fait l'objet de très nombreuses publications dans la presse locale et nationale, ainsi que 
dans les revues spécialisées - françaises et étrangères -, tant en architecture qu'en art sacré. Les articles consacrés au couvent, dès le début de l'année 1956, sont élogieux. Deux ans après, en 1958, l'opinion est toujours favorable à cette réalisation : une vue partielle de cette construction est choisie pour figurer au pavillon français de l'Exposition de Bruxelles, et le couvent fait partie des quatre œuvres françaises sélectionnées pour l'Exposition internationale d'art sacré de Louvain.

Les événements de 1968 et la mutation de la société qui a suivi ont largement contribué à vider les couvents, principalement les noviciats, en France. Le père gardien ne souhaitait pas que le lieu soit abandonné, alors même que la communauté religieuse remboursait encore des prêts que leur avaient accordés certains particuliers au moment de la construction. Il a donc proposé une alternative à la Province : transformer le couvent en centre ouvert à des formations religieuses. C'est la reconversion qui a été choisie également pour les couvents des dominicains de Lille et d'Eveux. C'est toujours l'activité du couvent de La Clarté-Dieu, qui a tissé un réseau d'échange avec l'évêché d'Évry.

\section{Description}

\section{Le plan}

Le couvent de La Clarté-Dieu est implanté dans un parc d'un peu moins de 5 hectares, sur le territoire de la commune d'Orsay. Le parc, boisé et agrémenté d'une pièce d'eau, présente une déclivité importante. Cette déclivité a été utilisée pour implanter les bâtiments.

Figure 4

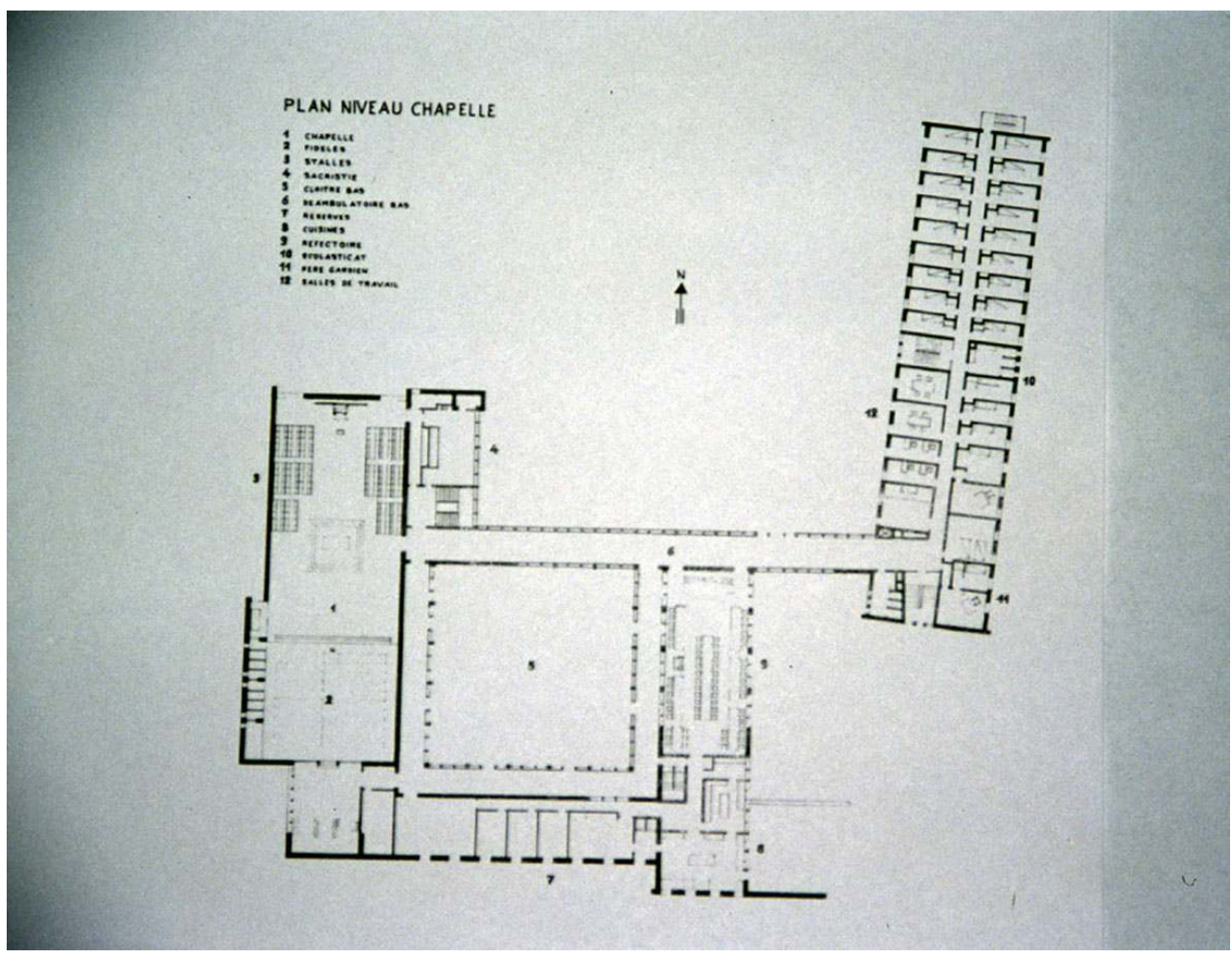

Plan de l'édifice (fonds d'archives de La Clarté-Dieu). Repro. Annabel Rémy, 2008.

(C) Annabel Rémy. 

les pièces (parloirs, infirmerie) et accès (vers la chapelle) accessibles au public, hors clôture. La clôture était «matérialisée » dans le hall par une porterie et un carrelage différencié, dissociant les espaces accessibles à tous des espaces réservés aux frères.

L'aile ouest est réservée à la chapelle (fig. $\mathbf{n}^{\circ} 5$ ), qui n'est pas orientée, et dont le chevet est au nord. Une tribune haute est accessible depuis le hall d'entrée, de même que le niveau bas qui est desservi par un escalier descendant du hall. Une sacristie surmontée du clocher (fig. $\mathbf{n}^{\circ} \mathbf{6}$ ) s'ouvre au nord-est de la chapelle, tandis qu'une crypte - prévue à l'origine pour être une salle capitulaire - est implantée sous la moitié nord de l'église.

Figure 5

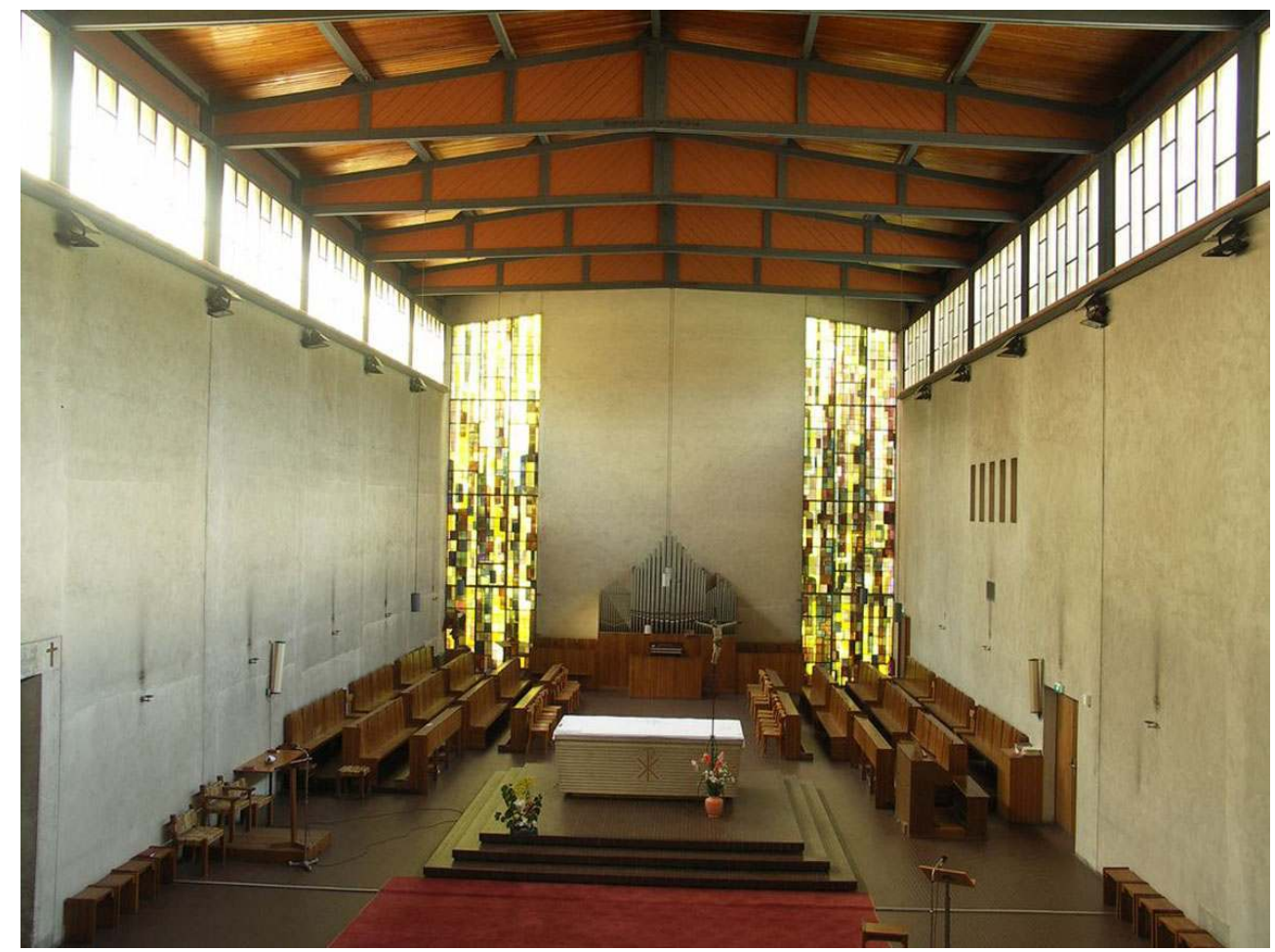

Chapelle, vue d'ensemble depuis la tribune. Phot. Annabel Rémy, 2008.

(C) Annabel Rémy. 
Figure 6

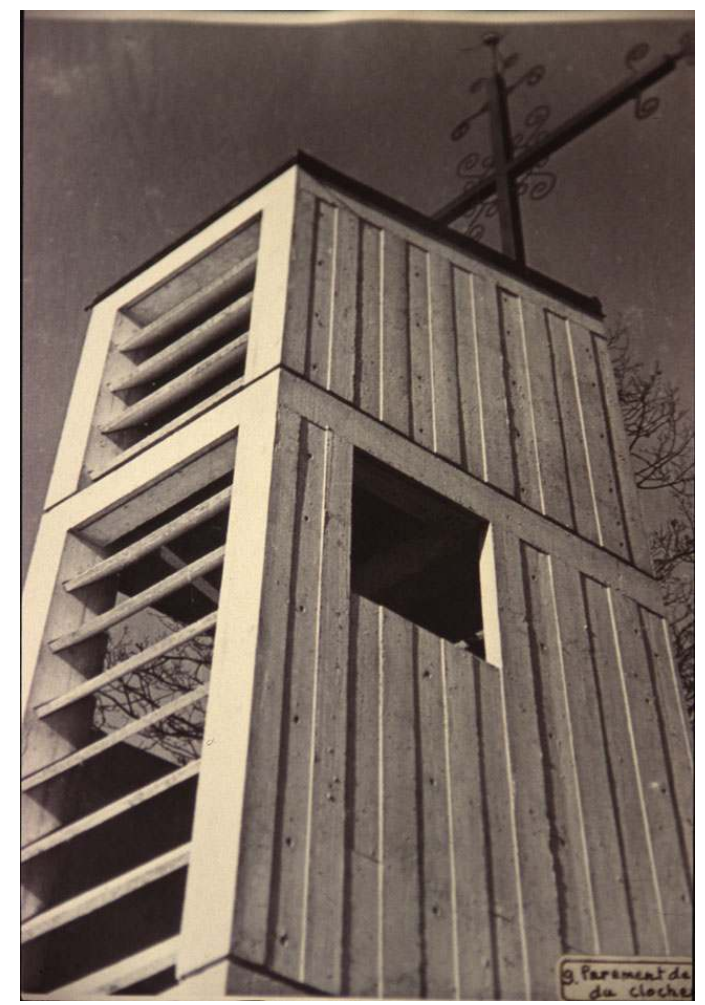

Le clocher (fonds Pascal Arsène-Henry). Repro. Annabel Rémy, 2008.

(C) Annabel Rémy.

19 L'aile est du quadrilatère est occupée en partie haute par des salles de classe et de réunion, au niveau inférieur (niveau du cloître à ciel ouvert et de la chapelle) par les cuisines et le réfectoire (fig. $n^{\circ}$ ) ), et enfin au-dessous par les ateliers et les oratoires (afin que chaque prêtre ordonné puisse dire la messe, le couvent ayant été conçu avant les réformes apportées par Vatican II).

Le cloître (fig. $\mathbf{n}^{\circ} \mathbf{8}$ ) est au centre de ces bâtiments (cloître haut fermé, et cloître bas à ciel ouvert), bordé d'un déambulatoire reliant les trois ailes précitées et fermant le quatrième côté au nord. L'extension de ce bras de déambulatoire nord relie le scolasticat, qui abrite les cellules des pères et des frères (à des étages différents), les sanitaires et douches, ainsi que la bibliothèque. 
Figure 7

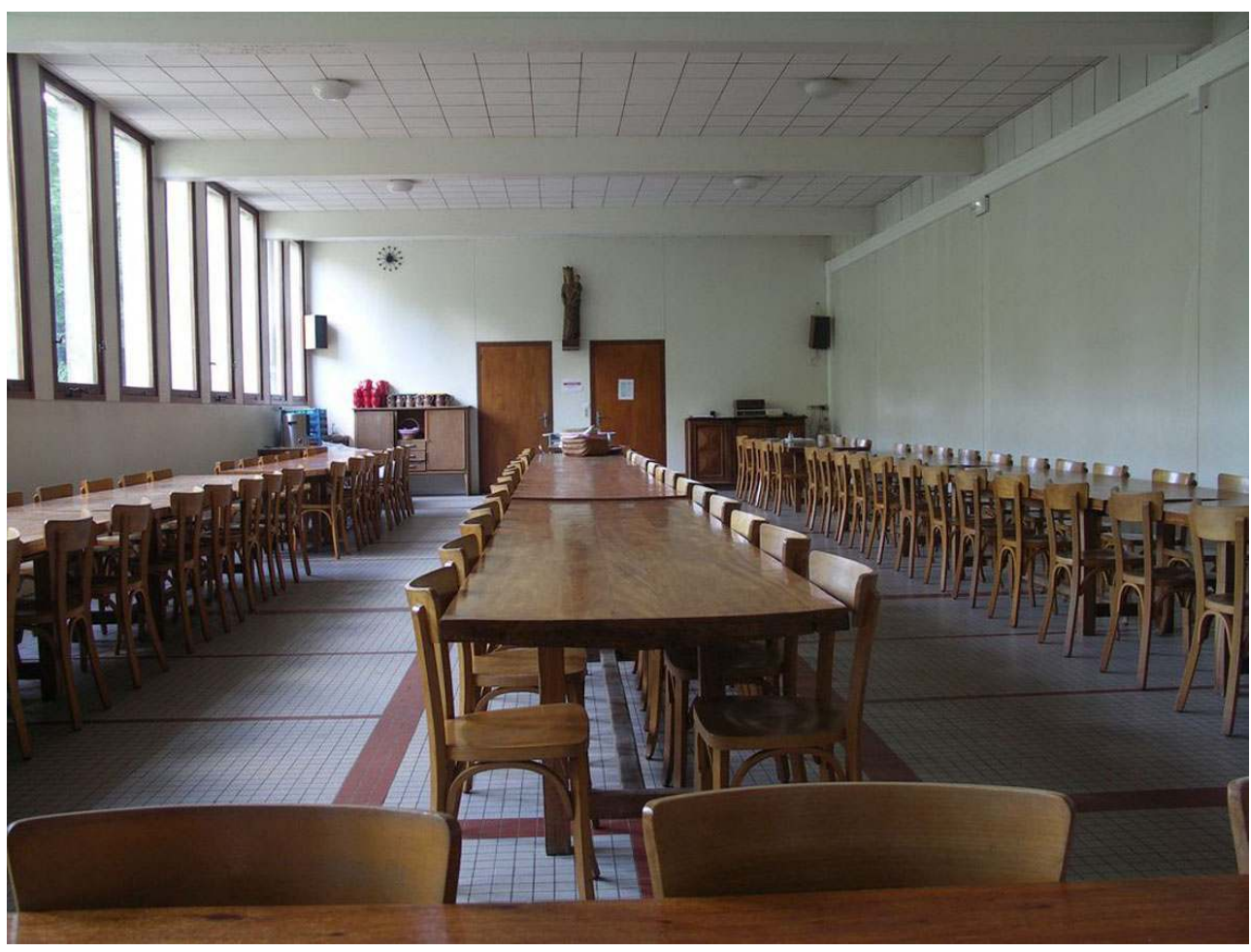

Le réfectoire. Phot. Annabel Rémy, 2008.

(c) Annabel Rémy. 
Figure 8

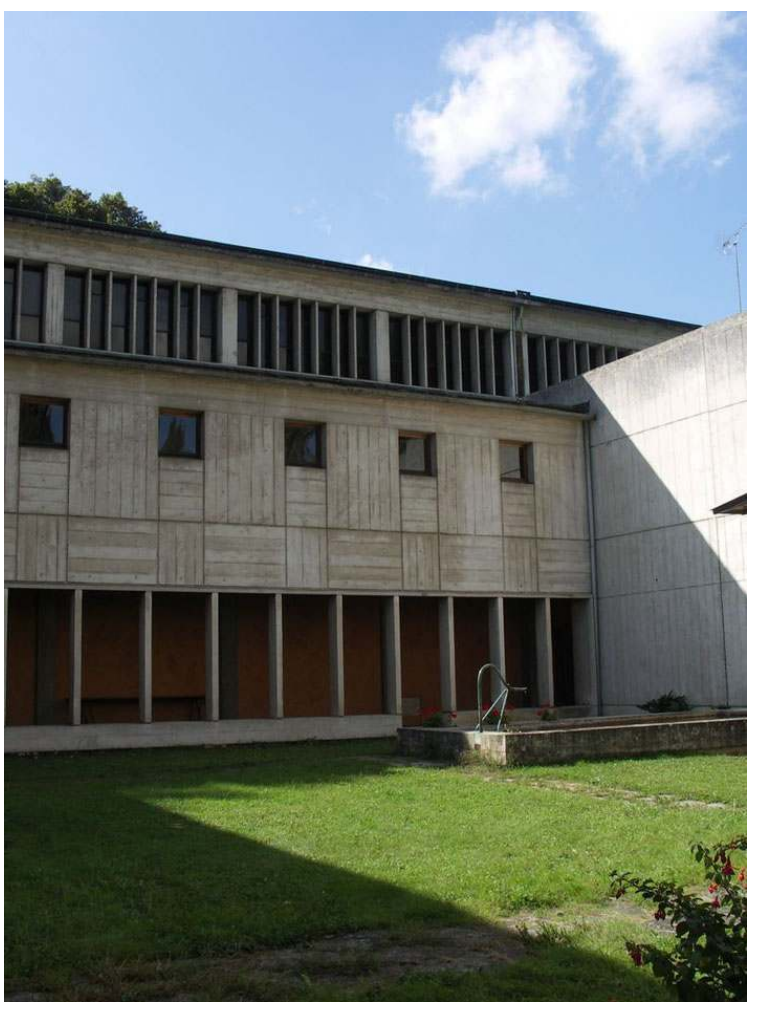

Le cloître. Phot. Annabel Rémy, 2008.

(C) Annabel Rémy.

\section{Les façades et toitures}




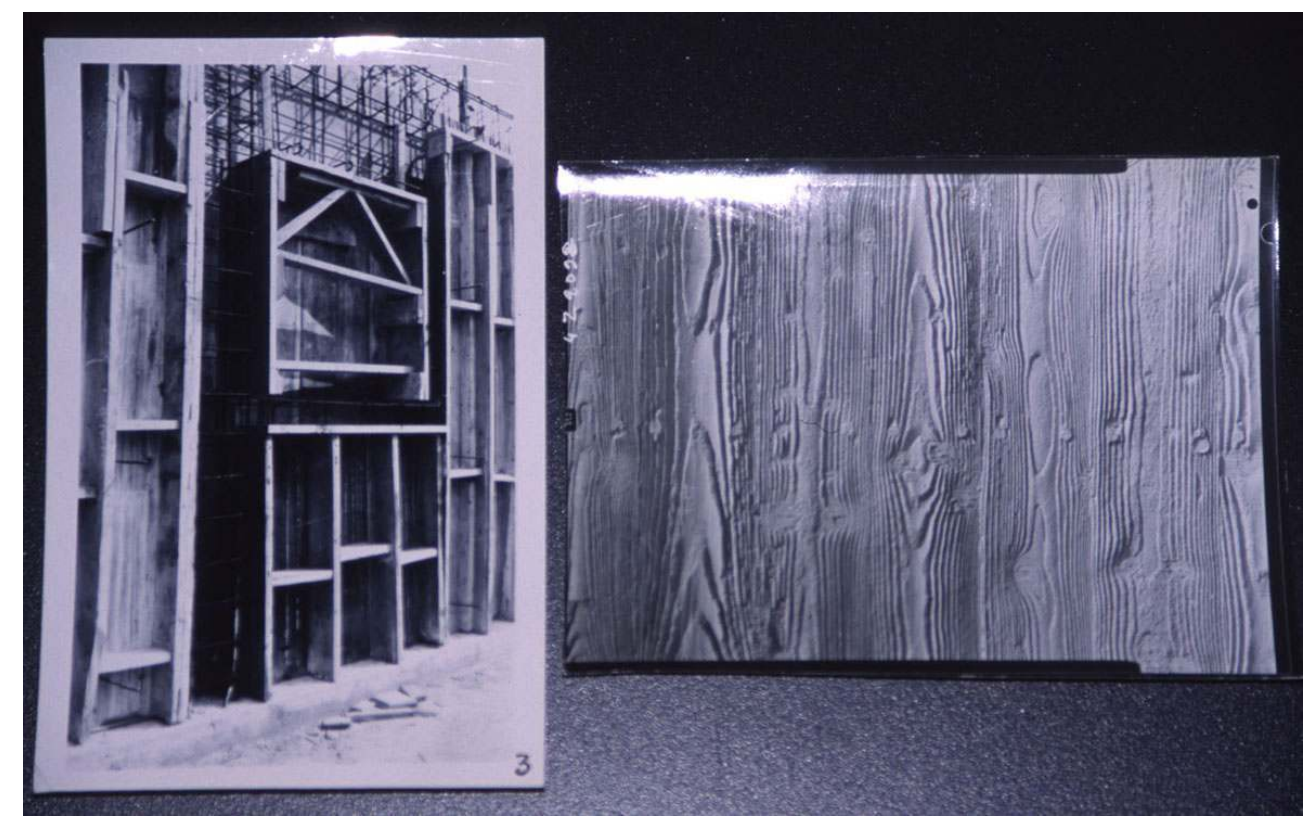

Coffrages (fonds d'archives de La Clarté-Dieu). Repro. Annabel Rémy, 2008.

(c) Annabel Rémy.

21 Les murs verticaux extérieurs sont constitués par des voiles de béton armé, de $8 \mathrm{~cm}$ d'épaisseur, raidis et laissés bruts de décoffrage. Les parements extérieurs sont réalisés à l'aide de coffrages en planches (fig. $\mathbf{n}^{\circ}$ 9) dont la dimension a été déterminée en vue d'un remploi d'environ 12 fois. Les coffrages sont réalisés en planches dressées et rabotées sur la face en contact avec les bétons et sur leurs rives. Les planches sont décalées en profondeur de $0,3 \mathrm{~cm}$ les unes par rapport aux autres, créant ainsi des jeux d'ombre et de lumière sur les façades. Les fenêtres, portes et pans de murs rythment les façades selon un multiple de $30 \mathrm{~cm}$ (en largeur): fenêtre de $60 \mathrm{~cm}$, pan de mur de $30 \mathrm{~cm}$, porte de $90 \mathrm{~cm}$, etc...

L'isolation thermique des murs de façade est assurée par un doublage en parpaings creux de ciment, ménageant un vide d'air isotherme de $3 \mathrm{~cm}$ entre le voile de béton armé des façades et le mur enduit intérieurement de mortier de chaux. La première dalle est coulée sur des panneaux de contre-plaqué permettant aux plafonds de rester en béton brut (ni enduit, ni plâtre). La totalité des trous et trémies pour le passage des canalisations a été prévue au moyen de taquets fixés dans les coffrages et retirés au décoffrage, ce qui permet une économie de temps et de moyens (principalement en main d'œuvre). Il en a été de même pour les points de fixation des huisseries, sanitaires et équipements sanitaires.

Une seule essence de bois a été utilisée pour les menuiseries extérieures, le niangon vert, bois exotique de couleur brun-rouge, huilé pour assurer au mieux sa préservation et son bon vieillissement. Il présente néanmoins l'inconvénient de se gauchir lorsqu'il est exposé à une forte humidité, phénomène observé sur plusieurs ouvertures du bâtiment.

La couverture est faite en feuilles de cuivre de $6 / 10^{\mathrm{e}}$ de millimètre posées à joints de bout sur un voligeage de pin avec interposition d'un feutre bitumeux pour une libre dilatation. L'isolation thermique est assurée par une couche de laine de verre posée sur la dalle de 
béton constituant le plafond du dernier niveau. La toiture du scolasticat, restaurée puis complètement reprise en 1966 et 1988, est faite d'un revêtement bitumeux revêtu d'une finition « vert ardoisine » pour assurer une harmonie visuelle avec le reste des toitures.

Une sculpture métallique de Costa Coulentianos (1918-1995) surmonte la porte d'entrée : elle figure deux anges entourant la croix.

\section{Les intérieurs}

La recherche de sobriété dans les décors se retrouve à l'intérieur, jusque dans les moindres détails. Ainsi, les pourtours d'huisserie, et de façon générale toute liaison entre deux matériaux distincts, sont traités par un creux d'un centimètre (environ) de large et de profondeur, dissimulant les fissures qui ne manquent pas d'apparaitre. Ce creux permet d'éviter l'usage de baguettes de finition.

Les murs sont recouverts, sauf dans les salles d'eau et cuisines, d'un simple enduit clair (blanc cassé). Les sols sont en carreaux de grès cérame de 5 centimètres de côté. Colorés en brun, beige ou gris, ils sont appareillés en motifs sommaires et discrets. Les plinthes, réalisées elles aussi en carreaux de grès cérame, sont intégrées dans le prolongement de l'enduit mural, ne présentant aucun ressaut (ce qui évite tout empoussièrement). Les sanitaires, salles d'eau et cuisines sont les seules pièces à être entièrement peintes, en bleu ou vert pâle. Les huisseries intérieures ont été posées après les enduits grâce à un système de gabarits métalliques réservant les feuillures dans les distributions et maintenant les taquets de fixation.

Le mobilier en place est celui conçu dès l'origine pour le couvent.

Le chauffage est assuré par une chaufferie fonctionnant au mazout et distribuant de l'eau à basse température dans les grilles déployées dans les dalles de plancher.

La chapelle est un vaste rectangle qui occupe toute l'aile ouest du couvent. Le décor intérieur a fait l'objet d'un effort particulier. Là aussi cependant, les murs sont traités en simple enduit clair, et les sols en carreaux de grès cérame de couleur brune. La charpente de la chapelle, composée de fermes en planches clouées, est laissée apparente.

Deux grandes verrières filtrantes au chevet de la chapelle (fig. $\mathbf{n}^{\circ} \mathbf{1 0}$ ) ainsi qu'une verrière filtrante dans la chapelle du Saint-Sacrement sont des compositions abstraites d'éléments de verre coloré assemblés par les peintres verriers Henri Déchanet et Paul Virilio (qui ont travaillé avec Léon Zack [1892-1980] pour l'église Notre-Dame-des-Pauvres à Issy-lesMoulineaux), selon des cartons du peintre Serge Rezvani (1928- ). Les deux grandes verrières adoptent des teintes assez douces de brun, jaune, mauve, tandis que la petite verrière du Saint-Sacrement est réalisée en teintes plus vives. La chapelle est également éclairée en partie haute par de petites fenêtres rectangulaires pourvues de verre transparent. 
Figure 10

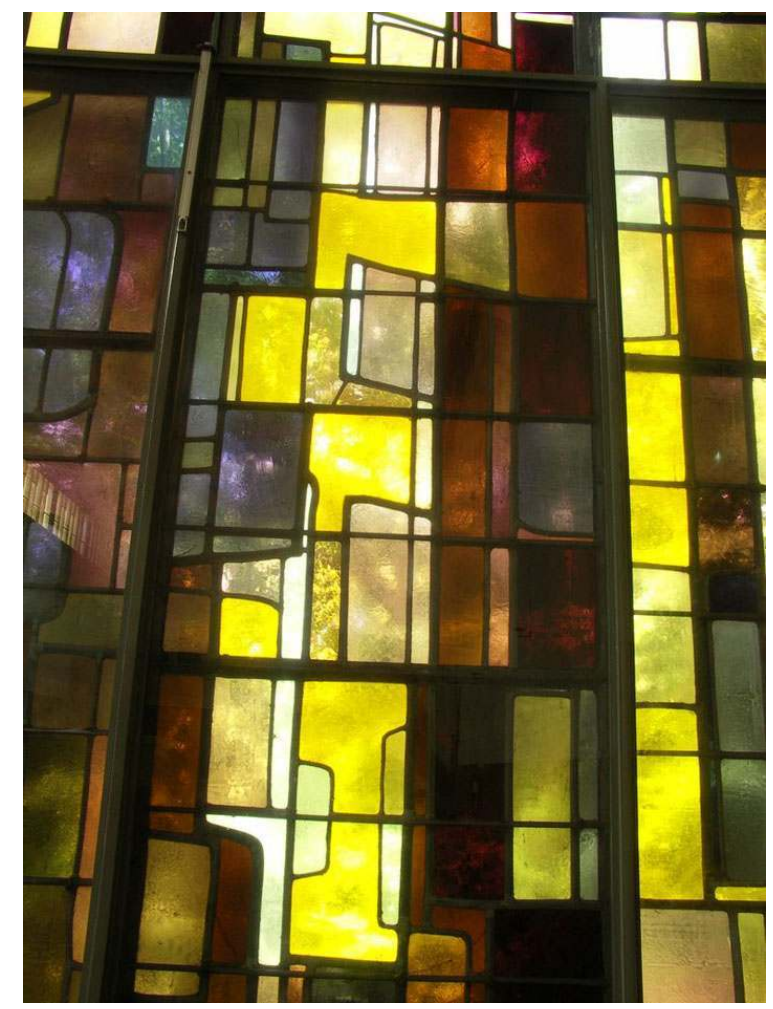

Détail d'une des grandes verrières de la chapelle, cartons de Serge Rezvani. Phot. Annabel Rémy, 2008.

(C) Annabel Rémy. 
Figure 11
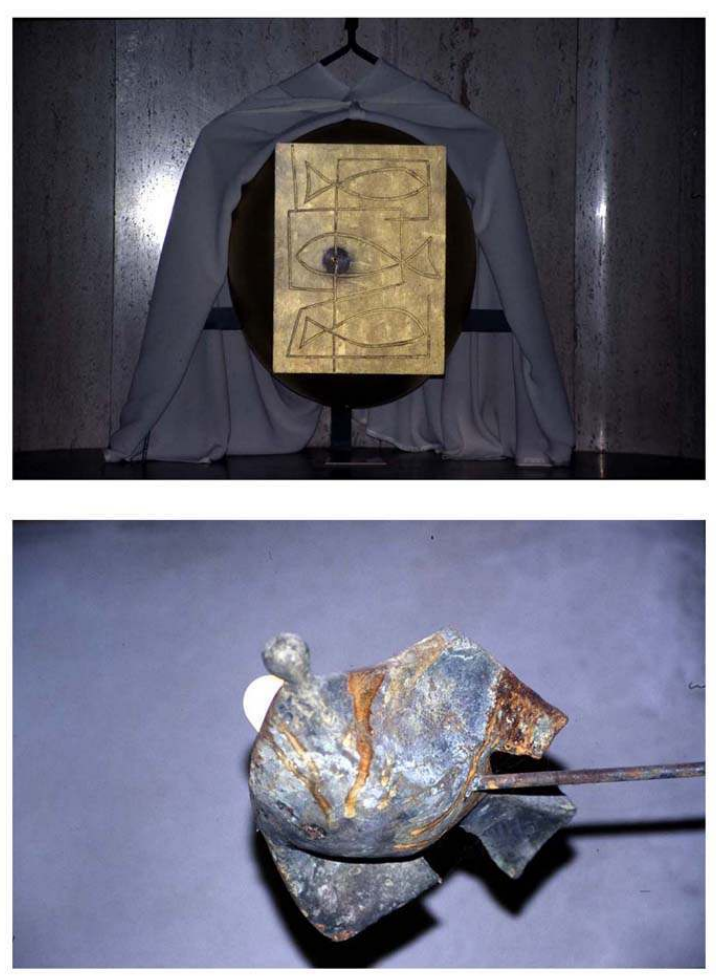

Le tabernacle, œuvre de Costa Coulentianos. Phot. Annabel Rémy, 2008.

(C) Annabel Rémy.

L'autel, situé sur une estrade carrelée de quatre marches, est en béton blanc moulé, orné d'un chrisme doré. Costa Coulentianos est l'auteur d'un ensemble d'objets dédiés au culte ainsi que du tabernacle (fig. $\mathbf{n}^{\circ} \mathbf{1 1}$ ). Les bancs des frères, ceux du public ainsi que les confessionnaux sont l'œuvre de Denis Voisin.

La couleur a été employée dans le cloître bas, ainsi que pour le lavabo rituel dans le déambulatoire. Ce sont là les seules couleurs vives, en dehors des verrières colorées de la chapelle, employées au sein du couvent. Elles soulignent l'importance majeure des parties de bâtiment ainsi colorées. Les plafonds du cloitre bas sont traités en teinte gris-noir, tandis que les murs sont en ocre-rouille (fig. $\mathbf{n}^{\circ} \mathbf{1 2}$ ). Le lavabo rituel (fig. $\left.\mathbf{n}^{\circ} \mathbf{1 3}\right)$ situé dans le déambulatoire, à l'entrée du réfectoire, est l'œuvre du peintre Jean-Claude Vignes (1924-1996). Une auge de plaques d'ardoise revêtues intérieurement de plomb est surmontée d'une mosaïque abstraite de petits carreaux de grès coloré et d'une plaque d'ardoise gravée, portant une inscription liturgique (inscription en ancien italien parlé au temps de saint François). 
Figure 12

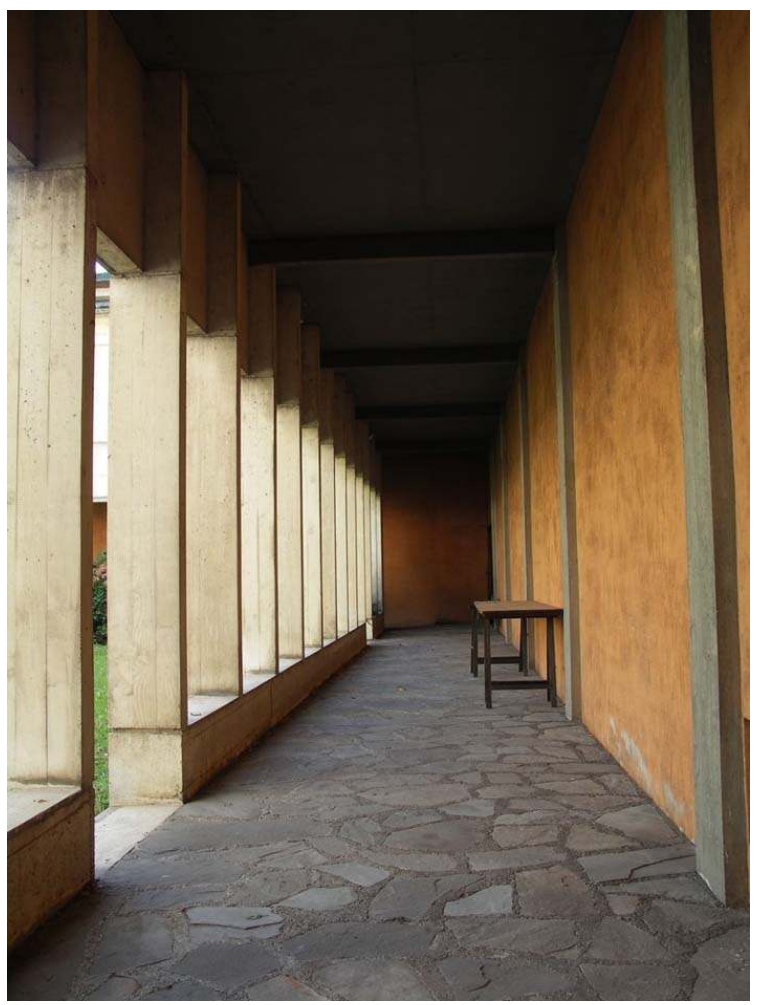

Couleurs des murs et du plafond du cloître bas. Phot. Annabel Rémy, 2008. (C) Annabel Rémy. 
Figure 13

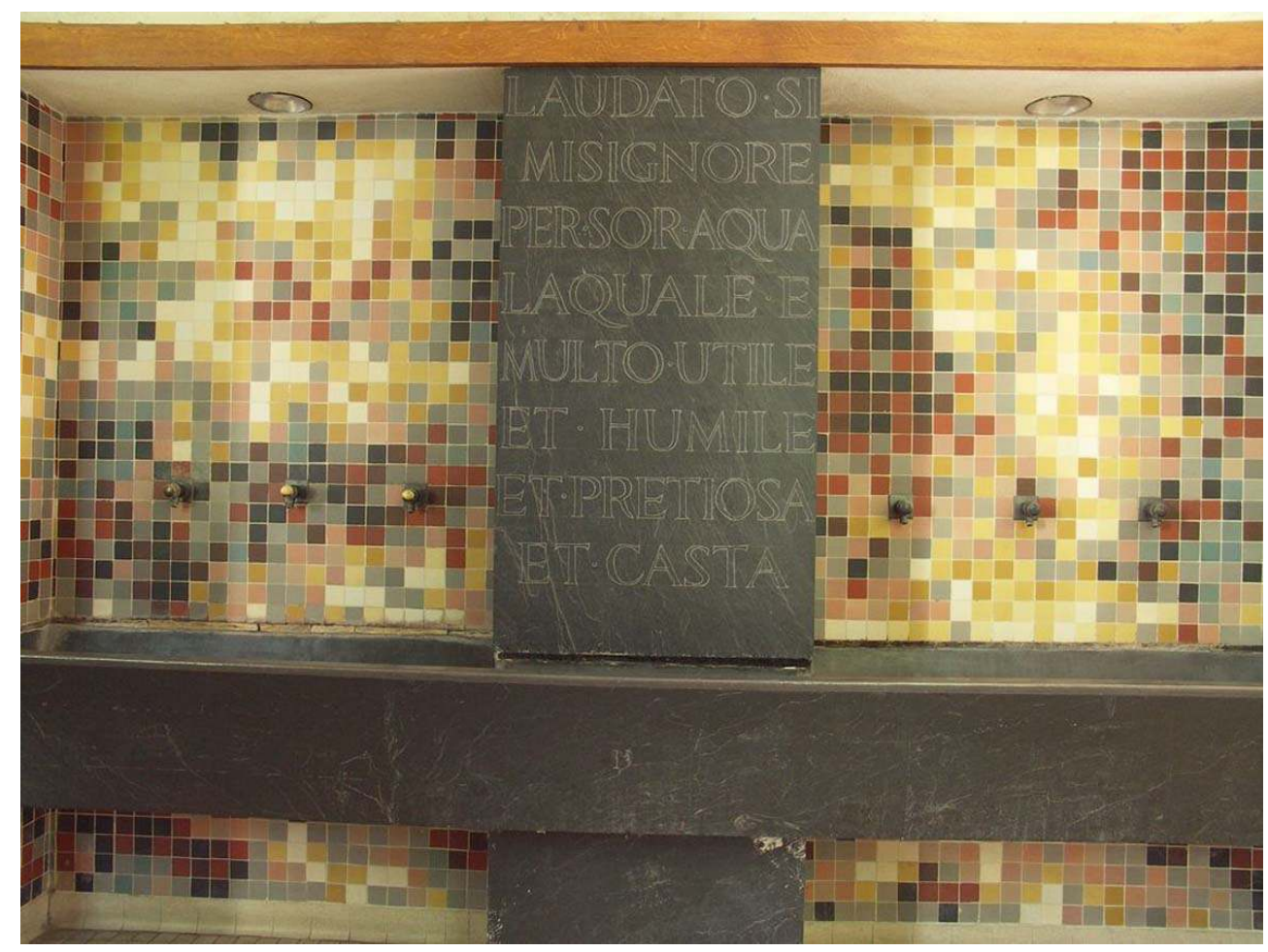

Lavabo rituel, œuvre de Jean-Claude Vignes. Phot. Annabel Rémy, 2008.

(C) Annabel Rémy.

34 Les cellules se répartissent de part et d'autre d'un couloir central dans l'aile du scolasticat. Des étages différents sont réservés aux pères et aux novices. Chaque cellule (fig. $\mathbf{n}^{\circ}{ }^{14}$ ) est un rectangle ouvert dans ses petits côtés par une porte d'un côté et une fenêtre de l'autre. Un petit cabinet de toilette (lave-pied) est séparé de la chambre par un pan de mur. Le point lumineux de la pièce est situé dans une ouverture au centre de ce mur, permettant à la fois d'éclairer la chambre et le cabinet de toilette. Une penderie fait face à ce cabinet. 
Figure 14

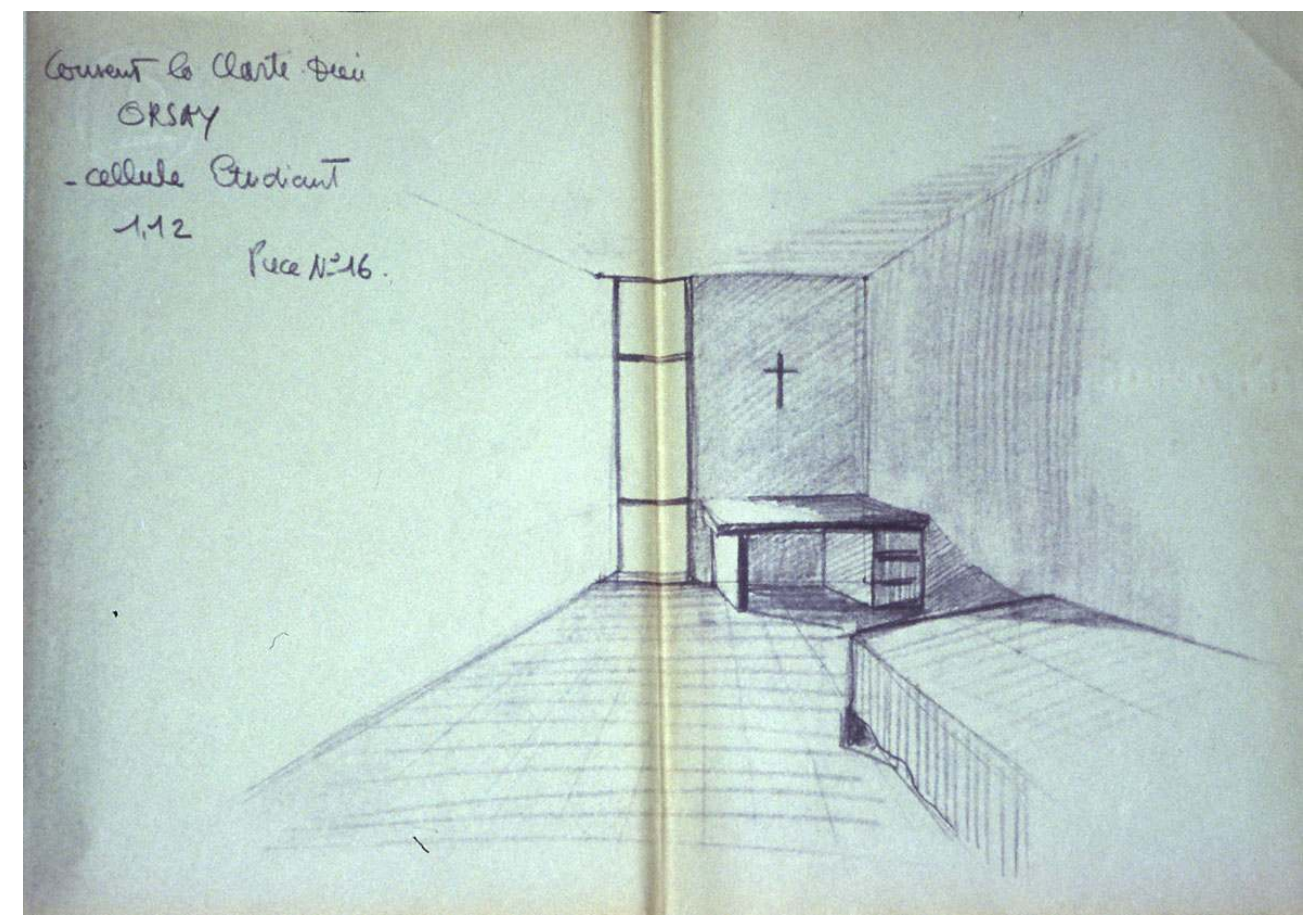

Dessin de l'aménagement d'une cellule (fonds d'archives de La Clarté-Dieu). Repro. Annabel Rémy, 2008.

(C) Annabel Rémy.

Le mobilier de la chambre est sommaire et identique pour toutes les cellules des novices. Il se compose d'un lit, d'une table de travail près de la fenêtre, d'une chaise, d'une étagère et d'un crucifix. Les pères disposent d'une surface de cellule plus grande, avec une autre table et d'autres chaises.

Le lien entre le passé et le présent est assuré, aux yeux des franciscains, par la présence de nombreuses sculptures anciennes en bois (du XIVe au XVIe siècles) : la Sainte-Trinité au portail d'entrée (très restaurée), la Vierge présentée dans la chapelle, et de nombreuses autres conservées dans les différentes salles du couvent.

\section{Les remaniements du bâtiment et de son parc}

Le bâtiment, dans son ensemble, a très bien vieilli et est actuellement dans un état sanitaire tout à fait satisfaisant. Le béton notamment est parfaitement conservé. Seul un éclatement existe sur l'escalier extérieur du scolasticat exposé au nord. Les arêtes des piliers du cloître sont restées très nettes. Ceci peut s'expliquer par les précautions dans la mise en œuvre des bétons lors du chantier.

Les $2500 \mathrm{~m}^{2}$ de toitures ont été la principale faiblesse du bâtiment. Les toitures ont été refaites en 1966 sur le bâtiment du scolasticat, non en cuivre, mais en revêtement bitumeux de couleur verte pour ne pas trancher esthétiquement avec les toitures de cuivre voisines. Elles l'ont été à nouveau en revêtement bitumeux en 1988 (ce qui correspond à un vieillissement normal du revêtement bitumeux choisi). 
39 Après 1968, la crypte a été transformée en salle de réunion, et les fenêtres ont été agrandies vers le bas de deux fois leur hauteur (1976-1977). Les cuisines ont été mises aux normes sanitaires. Les salles communes se sont révélées beaucoup trop sonores à l'usage, le béton laissé brut de décoffrage n'assurant pas une bonne acoustique. Les plafonds des salles de classe, des parloirs, du réfectoire et de la crypte ont donc été recouverts de dalles de polystyrène pour diminuer la résonance. La porterie et l'accueil ont été eux aussi transformés, en 1980, sous la conduite des frères Arsène-Henry, pour les mettre plus en accord avec les nouvelles fonctions du bâtiment. Le parc a été réduit par expropriation pour le passage de l'autoroute A10. En achetant le terrain, les franciscains savaient qu'une route était prévue dans un avenir proche sur une section limitrophe. D'une simple route départementale, le projet a en fait évolué vers une autoroute deux fois deux voies. Enfin, les paliers d'escalier ont été fermés par des murs de brique de verre et équipés de portes coupe-feu, tandis que des trappes de désenfumage ont été installées en 2004 dans les cages de trois des escaliers.

Des travaux d'aménagements sont toujours en cours, tant pour la mise aux normes d'un bâtiment accueillant du public que pour obtenir un plus grand confort des occupants. Il s'agit principalement de coffrage des plafonds dans le couloir fermé du cloître et le réfectoire (pour une meilleure isolation phonique) et de renouvellement des installations électriques. Si ces aménagements ne sont pas toujours esthétiques, au moins sont-ils réversibles. D'autres aménagements, plus lourds, sont à l'étude, telle l'augmentation de la surface du hall d'accueil par une grande verrière. Nécessaire pour les fonctions nouvelles du bâtiment, cette extension mériterait de faire l'objet d'une étude approfondie pour ne pas dénaturer le bâtiment.

\section{Une protection en suspens}

Un dossier de protection, en vue d'une inscription totale du bâtiment, a été présenté à la Commission régionale du patrimoine et des sites en juin 2005. Les membres de la Commission se sont prononcés à l'unanimité en faveur de cette protection, s'attachant au caractère exceptionnel de l'édifice, tant dans sa conception, dans l'innovation que représente sa réalisation en béton de ciment blanc, que dans son exceptionnelle conservation. Le propriétaire de l'édifice, l'Association des amis de La Clarté-Dieu, ayant exprimé par écrit son refus d'une inscription, le préfet de région n'a pas souhaité signer l'arrêté. L'édifice n'est donc toujours pas protégé au titre des Monuments historiques.

\section{NOTES}

1. Le programme, les notes préliminaires et l'ensemble des documents relatifs à la construction du couvent (plans, relations avec les entreprises, photographies du chantier, etc.) sont conservés dans les archives du couvent de La Clarté-Dieu conservées sur place. 
2. Archives consultées pour le couvent des dominicains de Lille, de l'architecte Pierre Pinsard, IFA (Institut Français d'architecture) cote : PINPI-52-02 et dossier de recensement conservé à la Médiathèque de l'architecture et du patrimoine. Voir dans la base Mérimée : notice PA59000088.

3. LE CORBUSIER. Un couvent de Le Corbusier, première publication en 1961, réédition des Éditions de Minuit, 2008. Voir dans la base Mérimée : notice PA00117761.

\section{RÉSUMÉS}

Édifié entre 1952 et 1956 par trois jeunes architectes, les frères Arsène-Henry et E. BesnardBernadac, le couvent de La Clarté-Dieu à Orsay (Essonne) est vraisemblablement le premier édifice en France construit en béton de ciment blanc dans une utilisation structurelle et non décorative. Un programme extraordinairement précis a été rédigé par deux frères franciscains. Il adopte les idées régulièrement développées par le père Régamey dans la revue l'Art Sacré, incitant à un renouveau puisant dans l'art et les techniques contemporaines. La conception des plans prit dix-huit mois, le chantier dura deux ans. Le couvent est implanté dans un parc d'un peu moins de 5 hectares. Ce parc boisé, agrémenté d'une pièce d'eau, présente une déclivité importante. Les murs verticaux extérieurs sont constitués par des voiles de béton armé, raidis et laissés bruts de décoffrage, créant des jeux de lumière et d'ombre sur les façades. La sobriété de la décoration du bâtiment est en accord avec celle de l'architecture. Inauguré le 4 juillet 1956, le couvent fait l'objet de très nombreuses publications dans la presse locale et nationale, ainsi que dans les revues spécialisées - françaises et étrangères -, tant en architecture qu'en art sacré

Built from 1952 to 1956 by three young architects, the Arsène-Henry brothers and E. BesnardBernadac, the La Clarté-Dieu convent in Orsay (Essonne, France) is probably the very first building made of a white cement concrete in a structural use and not a decorative one. An extraordinarily accurate program was written up by two franciscan brethen. It adopts the ideas regularly developed by the father Régamey in the journal l'Art Sacré, prompting to a revival borrowing from the contemporary art and technics. The design of the plans was made in 18 months, the construction lasted two years. The convent is located in a park of less than 5 hectares. This wooded park, which includes a pond, presents an important declivity. The vertical outside walls are composed of veils of reinforced concrete, stiffened and left rough, creating effects of light and shadow on the fronts. The soberness of the building adornment is concurring with the architecture one. Inaugurated on July the 4th, 1956, the convent was the object of very numerous publications in the local and national press, as well as in French or foreign magazines specialized in architecture or in sacred art 


\section{INDEX}

Mots-clés : entreprise Bachy, bureau d'étude Atbat, Orsay, Poissy, couvent de La Clarté-Dieu, noviciat de Champfleury, couvent des dominicains de Lille, couvent des dominicains d'Eveux, église Notre-Dame-des-Pauvres d'Issy-les-Moulineaux, frères Arsène-Henry, Xavier ArsèneHenry, Luc Arsène-Henry, Emmanuel Besnard-Bernadac, Marcel Page, Jacques Page, Pierre Vago, Novarina, Rémy Le Caisne, Dubuisson, Pierre Pinsard, Wladimir Bodiansky, Costa Coulentianos, Henri Déchanet, Paul Virilio, Léon Zack, Serge Rezvani, Jean-Claude Vignes, Denis Voisin, père Régamey, père Pol de Léon, père Brous, père Couturier, L'Art Sacré

\section{AUTEUR}

\section{ANNABEL RÉMY}

Régisseur d'œuvres au département des antiquités grecques, étrusques et romaines, musée du Louvre. annabel.remy@louvre.fr 